\title{
EVALUACIÓN DE LA EFICACIA, EFECTO RESIDUAL Y DE VOLTEO DE APLICACIONES EN PRETRASPLANTE DE INSECTICIDAS NICOTINOIDES Y MEZCLAS DE NICOTINOIDE-PIRETROIDE PARA EL CONTROL DE Myzus persicae nicotianae (HEMIPTERA: APHIDIDAE) EN TABACO
}

\author{
Evaluation of efficacy, residual and knock down effects of pretransplant applications \\ of nicotinoid and nicotinoid-pyrethroid insecticide mixtures for the control \\ of Myzus persicae nicotianae (Hemiptera: Aphididae) on tobacco
}

\author{
Eduardo Fuentes-Contreras ${ }^{1}$ *, Esteban Basoalto ${ }^{1}$, Claudio Sandoval ${ }^{1}$, Patricia Pavez ${ }^{1}$, \\ Claudia Leal' ${ }^{1}$, Raúl Burgos ${ }^{1}$ y Cristián Muñoz ${ }^{1}$
}

\begin{abstract}
A B S T R A C T
Pretransplant applications of imidacloprid, acetamiprid and thiamethoxam, and a mixture of imidacloprid and cyfluthrin were evaluated on tobacco (Nicotiana tabacum L.) seedbeds for the control of the aphid Myzus persicae nicotianae Blackman. Corrected mortality at $48 \mathrm{~h}$ after aphid release on plants in a seedbed was over $80 \%$ almost permanently up to 75 days after application (DAA) with a dose of $14 \mathrm{~g}$ a.i. $\mathrm{m}^{-2}$ seedbed of imidacloprid. In another experiment, the same level of corrected mortality was maintained by doses of $5.6 \mathrm{~g}$ a.i. $\mathrm{m}^{-2}$ seedbed of acetamiprid and $7 \mathrm{~g}$ a.i. $\mathrm{m}^{-2}$ seedbed of thiamethoxam up to 50 and 30 DAA, respectively. After 30 DAA for all the insecticides evaluated in both experiments, corrected mortality at $24 \mathrm{~h}$ was lower than at $48 \mathrm{~h}$ after aphid release on treated plants, which shows a limited knock down effect of these treatments. Finally, in another experiment a mixture of 10.5 and $0.21 \mathrm{~g}$ a.i. $\mathrm{m}^{-2}$ seedbed of imidacloprid and cyfluthrin, respectively, showed a good knock down effect, with corrected mortality higher than $80 \%$ up to 70 DAA at $48 \mathrm{~h}$ and without significant differences with corrected mortality at $24 \mathrm{~h}$ after aphid release on plants.
\end{abstract}

Key words: imidacloprid, thiamethoxam, acetamiprid, cyfluthrin.

\section{R E S U M E N}

Se evaluaron aplicaciones en pretrasplante en almacigueros de tabaco (Nicotiana tabacum L.) de imidacloprid, acetamiprid y tiametoxam, y una mezcla de imidacloprid y ciflutrina para el control del áfido Myzus persicae nicotianae Blackman. La dosis de imidacloprid de $14 \mathrm{~g}$ i.a. $\mathrm{m}^{-2}$ de almaciguero presentó mortalidades corregidas superiores al $80 \%$ casi permanentemente hasta los 75 días después de la aplicación (DDA) a las 48 h de la colocación de los áfidos sobre las plantas. En otro experimento acetamiprid en dosis de 5,6 g i.a. $\mathrm{m}^{-2}$ de almaciguero y tiametoxam en dosis de $7 \mathrm{~g}$ i.a. $\mathrm{m}^{-2}$ de almaciguero mantuvieron este mismo nivel de mortalidad corregida hasta los 50 y 30 DDA, respectivamente. A partir de los 30 DDA para todos los insecticidas evaluados en ambos experimentos, la mortalidad corregida a las $24 \mathrm{~h}$ fue menor que a las $48 \mathrm{~h}$ de la colocación de los áfidos sobre las plantas tratadas, lo cual muestra un limitado efecto de volteo de estos tratamientos. Finalmente, en otro experimento la mezcla de imidacloprid con ciflutrina en dosis de 10,5 y 0,21 g i.a. $\mathrm{m}^{-2}$ de almaciguero, respectivamente, presentó un buen efecto de volteo, con mortalidades corregidas superiores al $80 \%$ hasta los 70 DDA a las $48 \mathrm{~h}$ y sin diferencias significativas con la mortalidad corregida a las 24 h desde la colocación de los áfidos sobre las plantas.

Palabras clave: imidacloprid, tiametoxam, acetamiprid, ciflutrina.

\footnotetext{
${ }^{1}$ Universidad de Talca, Facultad de Ciencias Agrarias, Casilla 747, Talca, Chile. E-mail: efuentes@utalca.cl*Autor para correspondencia. Recibido: 22 de junio de 2005. Aceptado: 19 de agosto de 2005.
} 


\section{INTRODUCCIÓN}

El áfido verde del duraznero, Myzus persicae(Sulzer) es una plaga polífaga que causa daños económicos en varios cultivos hortofrutícolas. En el caso del cultivo del tabaco (Nicotiana tabacum L.) se ha descrito la presencia de una subespecie Myzus persicae nicotianae Blackman (Hemiptera: Aphididae) (Blackman e Eastop, 2006), la cual está presente en Chile desde hace aproximadamente una década (Fuentes-Contreras et al., 2004). La capacidad para desarrollar resistencia a los insecticidas es un atributo conocido de M. persicae (Unruh et al., 1996; Foster et al., 1998; 2002a; b; Mazzoni y Cravedi, 2002), que involucra también a la subespecie del tabaco (Koziol y Semtner, 1984; Harlow y Lampert, 1990; Wolf et al., 1994). Esta resistencia ha sido reportada para insecticidas organoclorados, organofosforados, carbamatos y piretroides (Devonshire et al., 1998; Casals y Silva, 1999; FuentesContreras et al., 2004), por lo que el manejo de esta plaga necesita orientarse en el marco de una estrategia de mitigación del desarrollo de resistencia a insecticidas, la cual se facilitaría con la incorporación de insecticidas de grupos químicos con nuevas modalidades de acción (Foster et al., 2002a; b; Nauen y Denholm, 2005).

Los insecticidas nicotinoides presentan una modalidad de acción diferente a la de los insecticidas tradicionales, la cual consiste en el bloqueo de la sinapsis nerviosa al unirse al receptor colinérgico de acetilcolina (Huang et al., 1999; Maienfisch et al., 2001). Muestran una muy buena actividad controladora de plagas picadoras-chupadoras, ya que tienen la capacidad de actuar tanto por contacto como por ingestión, al incorporarse desde el sustrato por las raíces y ser transportados por el xilema hasta los tejidos atacados por las plagas del follaje (Buchholz y Nauen, 2002; Maienfisch et al., 2001). Existen antecedentes que poblaciones de $M$. persicae resistentes a insecticidas tradicionales en varios cultivos, así como la subespecie del tabaco, pueden ser controladas efectivamente con estos insecticidas nicotinoides (Nauen et al., 1996; Foster et al., 2002a; Nauen y Elbert, 2003; Nauen y Denholm, 2005).

El objetivo del presente trabajo fue evaluar la eficacia, el efecto de volteo y el efecto residual de diferentes dosis de los insecticidas nicotinoides: imidacloprid, acetamiprid y tiametoxam, aplicados en tabaco en pretrasplante sobre $M$. persicae nicotianae. También se evaluaron distintas dosis de una mezcla de imidaclo- prid y ciflutrina en comparación con el organofosforado acefato.

\section{MATERIALES Y MÉTODOS}

Material vegetal. En los experimentos se utilizaron plantines de tabaco variedad Burley By64 (Profigen do Brasil Ltda.), los cuales se produjeron mediante el sistema de almacigueros "flotantes" descrito por Carrasco et al. (2000), siguiendo las recomendaciones técnicas de la Compañía Chilena de Tabacos.

Insectos. Los áfidos utilizados en los experimentos corresponden a la subespecie $M$. persicae nicotianae, según estudios morfométricos realizados con las claves de Blackman (1987). Éstos fueron recolectados en la temporada 1997-1998 en un campo de tabaco en la localidad de Requínoa, VI Región, siendo mantenidos en colonias bajo condiciones de temperatura $\left(25 \pm 1^{\circ} \mathrm{C}\right)$ y fotoperíodo (L16:O8) controlados en una cámara de cultivo (Conviron E4, Winnipeg, Canadá), sobre hojas de tabaco del mismo cultivar utilizado en los experimentos. De esta forma se mantuvieron individuos del mismo linaje asexual (clon) mediante reproducción asexual (partenogénesis) permanente. Este clon ha sido caracterizado como: i) anholocíclico, ii) con actividad de enzimas esterasas que le brindan un nivel de resistencia leve frente a organofosforados y carbamatos, y iii) con canales de sodio susceptibles a piretroides (Fuentes-Contreras et al., 2004).

Experimentos. Se realizaron tres experimentos en diferentes temporadas, los cuales se detallan a continuación. En el Experimento 1 (temporada 1999-2000) se evaluaron imidacloprid (Confidor $350 \mathrm{SC}$, Bayer AG, en concentración de $350 \mathrm{~g}$ i.a. $\left.\mathrm{L}^{-1}\right)$ en tres dosis de producto comercial: 15,30 y $40 \mathrm{~mL} \mathrm{~m}^{-2}$ almaciguero y un testigo sin aplicación. En el Experimento 2 (temporada 2002-2003) se evaluó acetamiprid (Mospilan 200 SP, Nippon Soda Co. Ltd., en concentración de $200 \mathrm{~g} \mathrm{~kg}^{-1}$ ) y tiametoxam (Actara $25 \mathrm{WG}$, Syngenta AG, en concentración de $\left.25 \mathrm{~g} \mathrm{~kg}^{-1}\right)$ en tres dosis de producto comercial: 7, 14 y $28 \mathrm{~g} \mathrm{~m}^{-2}$ almaciguero para ambos productos y un testigo sin aplicación. En el Experimento 3 (temporada 2000-2001) se evaluó la mezcla de imidacloprid y ciflutrina (Provado Supra 51 WP, Bayer AG, en concentración de 500 y $10 \mathrm{~g}$ i.a. $\mathrm{kg}^{-1}$, respectivamente) en cuatro dosis de producto comercial: $12,15,18 \mathrm{y}$ $21 \mathrm{~g} \mathrm{~m}^{-2}$ almaciguero. Además, se evaluó acefato (Orthene 75 SP, Tomen Corporation, en concentración de $750 \mathrm{~g}$ i.a. $\mathrm{kg}^{-1}$ ) en dosis de $24 \mathrm{~g} \mathrm{~m}^{-2}$ almaciguero de producto comercial y un testigo sin aplicación. 
Todos los experimentos se desarrollaron siguiendo el mismo protocolo de aplicación de los insecticidas. Dos días antes del trasplante, se realizaron las aplicaciones sobre los plantines de tabaco (15-20 cm de altura) en el almaciguero ( 242 celdillas, $25 \mathrm{~cm}^{3}$ por celdilla). Para esto se utilizó un aspersor manual, empleando un volumen de $500 \mathrm{~cm}^{3}$ por almaciguero. Los tratamientos testigo recibieron el mismo volumen de mojamiento solamente con agua. El trasplante se realizó dos días después a bolsas plásticas $\left(3.000 \mathrm{~cm}^{3}\right)$ con una mezcla 3:1 de suelo franco y tierra de hoja, cubriendo las plantas individualmente con mica y malla antiáfidos. Todas se mantuvieron en invernadero hasta el final de los experimentos respectivos.

Diseño experimental y análisis estadístico. En todos los experimentos se utilizó un diseño experimental completamente al azar, con las unidades experimentales ubicadas en un arreglo espacial aleatorio. En los Experimentos 1 y 2 se realizaron cinco repeticiones, y en el Experimento 3 se realizaron ocho repeticiones. En todos los casos las unidades experimentales estuvieron formadas por tres plantas cada una.

Para evaluar la eficacia de las dosis ensayadas para cada insecticida, se cuantificó la mortalidad de los áfidos provenientes de las crianzas de laboratorio, colocando grupos de 10 individuos ápteros adultos por cada planta para cada tratamiento (30 áfidos por unidad experimental). Con el propósito de medir el efecto residual de las dosis de los insecticidas ensayados se repitieron las evaluaciones periódicamente cada 20 días, desde los 15 a los 75 días después de la aplicación (DDA) para el experimento 1, mientras en los experi- mentos 2 y 3 se evaluó desde los 10 a los 70 DDA. Finalmente, para estimar el efecto de volteo de los insecticidas evaluados, se registró la mortalidad de los áfidos a las 24 y $48 \mathrm{~h}$ después de cada colocación de insectos, retirándose todos los individuos luego de esta evaluación y hasta una nueva colocación de áfidos. Todos los resultados se expresaron como mortalidad corregida (Abbott, 1925), la cual permite separar de la mortalidad observada el efecto de mortalidad natural presente en el respectivo tratamiento testigo.

Los datos de mortalidad corregida (MC) de cada experimento se analizaron por separado mediante ANDEVA. Los efectos principales fueron las dosis de los insecticidas utilizados, la fecha de evaluación (DDA) y el efecto de volteo (mortalidad a las 24 vs. 48 h), considerando esta última variable como un efecto de medida repetida en el tiempo. La separación de medias se realizó usando la prueba de comparación múltiple de Tukey con un nivel de confianza del 95\%. La MC se transformó a Arcsen $\sqrt{ } \mathrm{MC} / 100$ para cumplir los supuestos de homogeneidad de varianzas.

\section{RESULTADOS Y DISCUSIÓN}

En la primera evaluación correspondiente a los 15 DDA del Experimento 1 con imidacloprid, las dosis evaluadas no presentaron diferencias significativas entre sí (Cuadro 1). En la segunda, tercera y cuarta evaluación, se apreció un control significativamente mayor en la dosis más alta de imidacloprid (14 g i.a. $\mathrm{m}^{-2}$ almaciguero) en comparación con la dosis más baja (5,3 g i.a. $\mathrm{m}^{-2}$ almaciguero). La dosis de imidacloprid de 14 g i.a. $\mathrm{m}^{-2}$ de almaciguero presentó MC superio-

Cuadro 1. Mortalidad corregida de Abbot de Myzus persicae nicotianae con diferentes dosis de imidacloprid, aplicadas como tratamientos de pretrasplante sobre almacigueros de tabaco.

Table 1. Abbot's corrected mortality of Myzus persicae nicotianae, with different doses of imidacloprid applied as pretransplant treatments on tobacco seedbeds.

\begin{tabular}{|c|c|c|c|c|c|c|c|c|}
\hline \multirow[t]{3}{*}{ Tratamiento } & \multicolumn{8}{|c|}{ Mortalidad corregida (\%) } \\
\hline & \multicolumn{2}{|c|}{15 DDA $^{1}$} & \multicolumn{2}{|c|}{35 DDA } & \multicolumn{2}{|c|}{ 55 DDA } & \multicolumn{2}{|c|}{75 DDA } \\
\hline & $24 h^{2}$ & $48 \mathrm{~h}$ & $24 \mathrm{~h}$ & $48 \mathrm{~h}$ & $24 \mathrm{~h}$ & $48 \mathrm{~h}$ & $24 \mathrm{~h}$ & $48 \mathrm{~h}$ \\
\hline $\begin{array}{l}\text { Imidacloprid } \\
\qquad\left(5,3 \text { g i.a. } \mathrm{m}^{-2} \text { almaciguero }\right)\end{array}$ & $73,8 \mathrm{aB}$ & $97,5 \mathrm{aA}$ & $34,0 \mathrm{bBC}$ & $55,7 \mathrm{bB}$ & $27,1 \mathrm{aC}$ & $58,3 \mathrm{bB}$ & $21,3 \mathrm{aC}$ & $52,3 \mathrm{bB}$ \\
\hline $\begin{array}{l}\text { Imidacloprid } \\
\text { (10,5 g i.a. } \mathrm{m}^{-2} \text { almaciguero) }\end{array}$ & $77,1 \mathrm{aB}$ & 100 & $42,6 \mathrm{abC}$ & $81,1 \mathrm{aB}$ & $31,3 \mathrm{aC}$ & $73,8 \mathrm{abB}$ & $27,7 \mathrm{aC}$ & $75,3 \mathrm{abB}$ \\
\hline $\begin{array}{l}\text { Imidacloprid } \\
\text { (14 g i.a. } \mathrm{m}^{-2} \text { almaciguero) }\end{array}$ & $80,4 \mathrm{aB}$ & 100 & $59,6 \mathrm{aC}$ & $77,1 \mathrm{abB}$ & $39,6 \mathrm{aC}$ & $84,0 \mathrm{aB}$ & $29,8 \mathrm{aC}$ & $80,7 \mathrm{aB}$ \\
\hline
\end{tabular}

Diferentes letras minúsculas en columnas y mayúsculas en filas indican diferencias significativas según la prueba de Tukey $(\mathrm{P} \leq 0,05)$.

${ }^{1} \mathrm{DDA}=$ Días después de la aplicación.

${ }^{2} \mathrm{~h}=$ horas desde la colocación de los áfidos sobre las plantas tratadas. 
res al $80 \%$ casi permanentemente hasta los 75 DDA a las $48 \mathrm{~h}$ de la colocación de los áfidos sobre las plantas. En casi todas las evaluaciones y dosis ensayadas se observó una MC significativamente mayor a las 48 $\mathrm{h}$ en comparación con las $24 \mathrm{~h}$ (Cuadro 1).

En el Experimento 2 con acetamiprid y tiametoxam tampoco se presentaron diferencias significativas entre insecticidas y dosis evaluadas en la primera evaluación correspondiente a los 10 DDA (Cuadro 2). En la segunda evaluación correspondiente a los 30 DDA la MC fue significativamente mayor con las dosis más altas de acetamiprid (2,8 y 5,6 g i.a. $\mathrm{m}^{-2}$ almaciguero) y tiametoxam (3,5 y 7 g i.a. $\mathrm{m}^{-2}$ almaciguero), mientras en la tercera evaluación sólo la dosis mayor de acetamiprid mantuvo un efecto significativamente mejor que casi todos los tratamientos restantes (Cuadro 2). Finalmente, en la cuarta evaluación correspondiente a los 70 DDA, la MC no presentó diferencias significativas entre insecticidas y dosis evaluadas (Cuadro 2). En la mayoría de las evaluaciones y dosis ensayadas para cada insecticida se observó una MC significativamente mayor a las $48 \mathrm{~h}$ en comparación con las $24 \mathrm{~h}$ (Cuadro 2).

Los resultados de los Experimentos 1 y 2 evidenciaron un escaso efecto de volteo desde la segunda evaluación en adelante para todos los tratamientos ensayados. Un efecto residual con MC superiores al $80 \%$ durante la mayor parte del experimento fue observado con la dosis más alta de imidacloprid (14 g i.a. $\mathrm{m}^{-2}$ almaciguero) y hasta al menos los 30 DDA, con las dosis intermedias y altas de acetamiprid (2,8 y 5,6 g i.a. $\mathrm{m}^{-2}$ almaciguero) y tiametoxam $\left(3,5\right.$ y $7 \mathrm{~g}$ i.a. $\mathrm{m}^{-2}$ almaciguero). Este efecto residual relativamente largo de los insecticidas nicotinoides en tabaco, ha sido reportado también a nivel de campo en otros experimentos realizados con esta misma especie de áfido, incluyendo tanto aplicaciones pretrasplante como foliares postrasplante (Sannino et al., 2000a; b; McPherson et al., 2005). Una explicación de este fenómeno sería la adsorción de estos insecticidas a los coloides de materia orgánica del sustrato, desde donde son liberados progresivamente y absorbidos por las raíces durante un período más prolongado (Sarkar et al., 2001). El ingrediente activo es soluble en agua, siendo transportado por el xilema hasta las hojas sobre las cuales se alimentan los áfidos (Buchholz y Nauen, 2002; Laurent y Rathahao, 2003). En el caso del imidacloprid, se ha descrito que en el interior de la planta varios productos secundarios resultantes de la metabolización del ingrediente activo presentan una importante acción insecticida (Nauen et al., 1998b).

El limitado efecto de volteo que presentan los insecticidas neonicotinoides ha sido explicado debido a una acción antialimentaria sobre los áfidos, los cuales al ingerir la savia que contiene el insecticida detienen su

Cuadro 2. Mortalidad corregida de Abbot de Myzus persicae nicotianae con diferentes dosis de acetamiprid y tiametoxam, aplicadas como tratamientos de pretrasplante sobre almacigueros de tabaco.

Table 2. Abbot's corrected mortality of Myzus persicae nicotianae, with different doses of acetamiprid and thiamethoxam applied as pretransplant treatments on tobacco seedbeds.

\begin{tabular}{|c|c|c|c|c|c|c|c|c|}
\hline \multirow[t]{3}{*}{ Tratamiento } & \multicolumn{8}{|c|}{ Mortalidad corregida (\%) } \\
\hline & \multicolumn{2}{|c|}{$10 \mathrm{DDA}^{1}$} & \multicolumn{2}{|c|}{30 DDA } & \multicolumn{2}{|c|}{$50 \mathrm{DDA}$} & \multicolumn{2}{|c|}{70 DDA } \\
\hline & $24 h^{2}$ & $48 \mathrm{~h}$ & $24 \mathrm{~h}$ & $48 \mathrm{~h}$ & $24 \mathrm{~h}$ & $48 \mathrm{~h}$ & $24 \mathrm{~h}$ & $48 \mathrm{~h}$ \\
\hline $\begin{array}{l}\text { Acetamiprid } \\
\left(1,4 \mathrm{~g} \mathrm{~m}^{-2} \text { almaciguero }\right)\end{array}$ & $97,7 \mathrm{aA}$ & $100 \mathrm{aA}$ & $34,1 \mathrm{bBC}$ & $60,5 \mathrm{bB}$ & $28,6 \mathrm{abC}$ & $65,0 \mathrm{abB}$ & $17,2 \mathrm{aC}$ & $36,1 \mathrm{aBC}$ \\
\hline $\begin{array}{l}\text { Acetamiprid } \\
\left(2,8 \mathrm{~g} \mathrm{~m}^{-2} \text { almaciguero }\right)\end{array}$ & $88,4 \mathrm{aB}$ & $100 \mathrm{aA}$ & $61,0 \mathrm{abC}$ & $86,8 \mathrm{aB}$ & $23,8 \mathrm{bC}$ & $63,1 \mathrm{bBC}$ & $33,5 \mathrm{aC}$ & $50,0 \mathrm{aC}$ \\
\hline $\begin{array}{l}\text { Acetamiprid } \\
\left(5,6 \mathrm{~g} \mathrm{~m}^{-2} \text { almaciguero }\right)\end{array}$ & $86,0 \mathrm{aB}$ & $100 \mathrm{aA}$ & $68,3 \mathrm{aBC}$ & $92,1 \mathrm{aB}$ & $47,6 \mathrm{aC}$ & $88,6 \mathrm{aB}$ & $32,6 \mathrm{aC}$ & $44,4 \mathrm{aC}$ \\
\hline $\begin{array}{l}\text { Tiametoxam } \\
\qquad\left(1,75 \mathrm{~g} \mathrm{~m}^{-2} \text { almaciguero }\right)\end{array}$ & $90,7 \mathrm{aB}$ & $100 \mathrm{aA}$ & $34,1 \mathrm{bCD}$ & $57,9 \mathrm{bC}$ & $11,0 \mathrm{bD}$ & $56,7 \mathrm{bC}$ & $25,6 \mathrm{aD}$ & $44,4 \mathrm{aC}$ \\
\hline $\begin{array}{l}\text { Tiametoxam } \\
\left(3,5 \mathrm{~g} \mathrm{~m}^{-2} \text { almaciguero }\right)\end{array}$ & $90,7 \mathrm{aB}$ & $100 \mathrm{aA}$ & $61,0 \mathrm{abC}$ & $89,5 \mathrm{aB}$ & $20,5 \mathrm{bCD}$ & $38,9 \mathrm{bC}$ & $19,5 \mathrm{aD}$ & $36,1 \mathrm{aC}$ \\
\hline $\begin{array}{l}\text { Tiametoxam } \\
\left(7,0 \mathrm{~g} \mathrm{~m}^{-2} \text { almaciguero }\right)\end{array}$ & $79,1 \mathrm{aB}$ & $100 \mathrm{aA}$ & $70,7 \mathrm{aB}$ & $100 \mathrm{aA}$ & $31,0 \mathrm{abC}$ & $61,9 \mathrm{bC}$ & $27,9 \mathrm{aC}$ & $38,9 \mathrm{aC}$ \\
\hline
\end{tabular}

Diferentes letras minúsculas en columnas y mayúsculas en filas indican diferencias significativas según la prueba de Tukey $(\mathrm{P} \leq 0,05)$.

${ }^{1}$ DDA $=$ Días después de la aplicación.

$2 \mathrm{~h}=$ horas desde la colocación de los áfidos sobre las plantas tratadas. 
alimentación, provocando la muerte tanto por su efecto neurotóxico como por inanición y/o deshidratación (Devine et al., 1996; Nauen y Elbert, 1997; Nauen et al., 1998a).

En el Experimento 3 se puede observar que a partir de los 10 DDA y hasta los 70 DDA, la mortalidad corregida de las distintas dosis de imidacloprid y ciflutrina, evaluada a las 24 ó $48 \mathrm{~h}$, es significativamente y permanentemente superior a la del tratamiento estándar con acefato (Cuadro 3). En particular desde los 50 DDA, tanto para las evaluaciones a las 24 y $48 \mathrm{~h}$, las dosis de imidacloprid y ciflutrina de $9+0,18$ y $10,5+$ 0,21 g i.a. $\mathrm{m}^{-2}$ de almaciguero alcanzan las mayores eficacias en relación con la menor dosis evaluada $(6+$ 0,12 g i.a. $\mathrm{m}^{-2}$ almaciguero) de esta misma mezcla de productos (Cuadro 3).

El efecto de volteo de imidacloprid y ciflutrina se manifiesta especialmente en las dosis más altas utilizadas, siendo significativamente superior al observado para el tratamiento estándar con acefato. Estos datos sugieren que la incorporación del piretroide ciflutrina en una mezcla con imidacloprid podría mejorar el efecto de volteo de este producto nicotinoide sobre el áfido del tabaco.

La incorporación de ciflutrina en mezcla con imidacloprid, permite además, controlar plagas de lepidópteros que no son afectadas por los insecticidas nicotinoides ensayados en nuestro estudio (Leal, 2001). Tam- bién es importante mencionar que las aplicaciones pretrasplante pueden tener algunas ventajas sobre las aplicaciones foliares. Los insecticidas nicotinoides, al igual que la mayoría de los insecticidas tradicionales, no son particularmente selectivos sobre la mayor parte de la fauna benéfica del agroecosistema (Maienfisch et al., 2001). Por lo tanto, disminuir los residuos a los que se exponen los polinizadores y/o enemigos naturales sobre las hojas, reduce la toxicidad directa del insecticida sobre estos organismos benéficos (Vincent et al., 2000).

Por otra parte, la presencia sistémica de estos insecticidas y sus moléculas derivadas circulando en la planta, podría imponer un riesgo sobre los polinizadores y algunos enemigos naturales que se alimentan del néctar de las flores o de nectarios extraflorales. Sobre los polinizadores, entre ellos la abeja de miel (Apis mellifera L.) alimentándose en girasoles (Helianthus annus L.), no se han observado efectos negativos a nivel de campo (Schmuck et al., 2001). Para enemigos naturales se han encontrado efectos subletales, es decir de modificación de su conducta o disminución de su longevidad, pero solamente en especies que tienen dietas parcialmente fitófagas, alimentándose en forma importante de néctar o polen (Torres et al., 2003). En el caso particular del tabaco, la práctica agronómica de remoción de las inflorescencias para favorecer la maduración de las hojas, reduce considerablemente esta posibilidad de efecto indirecto del insecticida sobre la fauna benéfica.

Cuadro 3. Mortalidad corregida de Abbot de Myzus persicae nicotianae con diferentes dosis de imidacloprid + ciflutrina y acefato, aplicadas como tratamientos de pretrasplante sobre almacigueros de tabaco.

Table 3. Abbot's corrected mortality of Myzus persicae nicotianae, with different doses of imidacloprid + cyfluthrin and acephate applied as pretransplant treatments on tobacco seedbeds.

\begin{tabular}{|c|c|c|c|c|c|c|c|c|}
\hline \multirow[t]{3}{*}{ Tratamiento } & \multicolumn{8}{|c|}{ Mortalidad corregida (\%) } \\
\hline & \multicolumn{2}{|c|}{10 DDA $^{1}$} & \multicolumn{2}{|c|}{30 DDA } & \multicolumn{2}{|c|}{ 50 DDA } & \multicolumn{2}{|c|}{70 DDA } \\
\hline & $24 h^{2}$ & $48 \mathrm{~h}$ & $24 \mathrm{~h}$ & $48 \mathrm{~h}$ & $24 \mathrm{~h}$ & $48 \mathrm{~h}$ & $24 \mathrm{~h}$ & $48 \mathrm{~h}$ \\
\hline $\begin{array}{l}\text { Imidacloprid + ciflutrina } \\
\left(6+0,12 \text { g i.a. } \mathrm{m}^{-2} \text { almaciguero }\right)\end{array}$ & $85,5 \mathrm{bB}$ & $100 \mathrm{aA}$ & $53,8 \mathrm{cC}$ & $73,8 \mathrm{bB}$ & $73,1 \mathrm{bB}$ & $73,1 \mathrm{bB}$ & $42,5 \quad b C$ & $42,5 \mathrm{bC}$ \\
\hline $\begin{array}{l}\text { Imidacloprid + ciflutrina } \\
\quad\left(7,5+0,15 \text { g i.a. } \mathrm{m}^{-2} \text { almaciguero }\right)\end{array}$ & $87,9 \mathrm{bB}$ & 100 & $72,5 \mathrm{bcBC}$ & $87,5 \mathrm{abB}$ & $94,1 \mathrm{aB}$ & $94,1 \mathrm{aB}$ & $63,8 \mathrm{aC}$ & $63,8 \mathrm{aC}$ \\
\hline $\begin{array}{l}\text { Imidacloprid + ciflutrina } \\
\qquad\left(9+0,18 \text { g i.a. } \mathrm{m}^{-2} \text { almaciguero }\right)\end{array}$ & 100 & 100 & $82,3 \mathrm{abB}$ & $91,3 \mathrm{aB}$ & $93,2 \mathrm{aB}$ & $93,2 \mathrm{aB}$ & 81,3 aB & $81,3 \mathrm{aB}$ \\
\hline $\begin{array}{l}\text { Acefato } \\
\left(18 \text { g i.a. } \mathrm{m}^{-2} \text { almaciguero }\right)\end{array}$ & $56,4 \mathrm{cB}$ & $94,5 \mathrm{bA}$ & $7,5 \mathrm{dCD}$ & $16,3 \mathrm{cC}$ & $15,0 \mathrm{cC}$ & $17,3 \mathrm{cC}$ & $1,25 \mathrm{cD}$ & $2,5 \mathrm{cD}$ \\
\hline
\end{tabular}

Diferentes letras minúsculas en columnas y mayúsculas en filas indican diferencias significativas según la prueba de Tukey $(\mathrm{P} \leq 0,05)$.

${ }^{1} \mathrm{DDA}=$ Días después de la aplicación.

${ }^{2} \mathrm{~h}=$ horas desde la colocación de los áfidos sobre las plantas tratadas. 


\section{CONCLUSIONES}

1.- La dosis de imidacloprid de $14 \mathrm{~g}$ i.a. $\mathrm{m}^{-2}$ de almaciguero presentó significativamente mejor eficacia que las otras dosis evaluadas de este insecticida, con MC superiores al $80 \%$ casi permanentemente hasta los 75 DDA a las 48 h de la colocación de los áfidos sobre las plantas.

2.- Todas las dosis evaluadas de imidacloprid presentaron un reducido efecto de volteo con diferencias significativas de $\mathrm{MC}$ entre las 24 y $48 \mathrm{~h}$ de la colocación de los áfidos sobre las plantas.

3.- Acetamiprid en dosis de 5,6 g i.a. $\mathrm{m}^{-2}$ de almaciguero y tiametoxam en dosis de $7 \mathrm{~g}$ i.a. $\mathrm{m}^{-2}$ de almaciguero presentaron significativamente mayor eficacia que las otras dosis evaluadas, con MC superiores a $80 \%$ hasta los 50 y 30 DDA a las 48 h de la colocación de los áfidos sobre las plantas respectivamente.

4.- Todas las dosis evaluadas de acetamiprid y tiametoxam presentaron un reducido efecto de volteo con diferencias significativas de MC entre las 24 y $48 \mathrm{~h}$ de la colocación de los áfidos sobre las plantas.

5.- La mezcla de imidacloprid con ciflutrina en dosis de 10,5 y 0,21 g i.a. $\mathrm{m}^{-2}$ de almaciguero respectivamente, presentó $\mathrm{MC}$ superiores al $80 \%$ hasta los 70 DDA a las $48 \mathrm{~h}$ desde la colocación de los áfidos sobre las plantas.

6.- La mezcla de imidacloprid con ciflutrina en dosis de 10,5 y 0,21 g i.a. $\mathrm{m}^{-2}$ de almaciguero respectivamente, presentó un efecto de volteo sin diferencias significativas de $\mathrm{MC}$ entre las 24 y 48 h de la colocación de los áfidos sobre las plantas.

\section{RECONOCIMIENTOS}

Agradecemos el financiamiento de FONDECYT (Proyecto 1000079) y de Bayer CropScience de Chile, como asimismo la colaboración y facilidades otorgadas por Chiletabacos, Syngenta y Bayer de Chile. Se agradecen también los valiosos aportes realizados por el editor y los árbitros anónimos.

\section{LITERATURA CITADA}

Abbott, W.S. 1925. A method of computing the effectiveness of an insecticide. J. Econ. Entomol. 18:265-267.

Blackman, R.L. 1987. Morphological discrimination of a tobacco-feeding form from Myzus persicae (Sulzer) (Hemiptera: Aphididae), and a key to New World Myzus (Nectarosiphon) species. Bull. Entomol. Res. 77:713-730.

Blackman, R.L., and V.F. Eastop. 2007. Taxonomic issues. H.F. van Emden and R. Harrington (eds.). Aphids as crop pests. CABI Publishing, Wallingford, UK. (In press)

Buchholz, A., and R. Nauen. 2002. Translocation and translaminar bioavailability of two neonicotinoid insecticides after foliar application to cabbage and cotton. Pest Manage. Sci. 58:10-16.

Carrasco, G., P. Rebolledo, and P. Valverde. 2000. Floating system: an alternative for producing tobacco transplants in Chile. Acta Hortic. 517:241-246.

Casals, P., y G. Silva. 1999. Susceptibilidad a insecticidas del pulgón verde del duraznero (Myzus persicae Sulzer) en remolacha. Agrociencia (Chile) 15:55-61.

Devine, G.J., Z.K. Harking, A.W. Scarr, and A.L. Devonshire. 1996. Lethal and sublethal effects of imidacloprid on nicotine-tolerant Myzus nicotianae and Myzus persicae. Pestic. Sci. 48:57-62.
Devonshire, A.L., L.M. Field, S.P. Foster, G.D. Moores, M.S. Williamson, and R.L. Blackman. 1998. The evolution of insecticide resistance in the peach-potato aphid, Myzus persicae. Philos. Trans. R. Soc. London, Ser. B 353:1677-1684.

Foster, S.P., I. Denholm, Z.K. Harling, G.D Moores, and A.L. Devonshire. 1998. Intensification of insecticide resistance in UK field populations of the peach-potato aphid, Myzus persicae (Hemiptera: Aphididae) in 1996. Bull. Entomol. Res. 88:127-130.

Foster, S.P., I. Denholm, and R. Thompson. 2002a. Variation in response to neonicotinoid insecticides in peach-potato aphids, Myzus persicae (Hemiptera: Aphididae). Pest Manage. Sci. 59:166-173.

Foster, S.P., R. Harrington, A.M. Dewar, I. Denholm, and A.L. Devonshire. 2002b. Temporal and spatial dynamics of insecticide resistance in Myzus persicae (Hemiptera: Aphididae). Pest Manage. Sci. 58:895907.

Fuentes-Contreras, E., C.C. Figueroa, M. Reyes, L.M. Briones, and H.M. Niemeyer. 2004. Genetic diversity and insecticide resistance of Myzus persicae (Hemiptera: Aphididae) populations from tobacco in Chile: evidence for the existence of a single predominant clone. Bull. Entomol. Res. 94:11-18. 
Harlow, C.D., and E.P. Lampert. 1990. Resistance mechanisms in two color forms of the tobacco aphid (Homoptera: Aphididae). J. Econ. Entomol. 83:21302135.

Huang, Y., M.S. Williamson, A.L. Devonshire, J.D. Windass, S.J. Lansdell, and N.S. Millar. 1999. Molecular characterization and imidacloprid selectivity of nicotinic acetylcholine receptor subunits from the peach-potato aphid Myzus persicae. J. Neurochem. 73:380-389.

Koziol, F.S., and P.J. Semtner. 1984. Extent of resistance to organophosphorous insecticides in field populations of the green peach aphid (Homoptera: Aphididae) infesting flue-cured tobacco in Virginia. J. Econ. Entomol. 77:1-3.

Laurent, F.M., and E. Rathahao. 2003. Distribution of [C-14]imidacloprid in sunflowers (Helianthus annuus L.) following seed treatment. J. Agric. Food Chem. 51: 8005-8010.

Leal, R.S. 2001. The use of Confidor ${ }^{\circledR} \mathrm{S}$ in the float, a new tobacco seedlings production system in the south of Brazil. Planzenschutz-Nachr. Bayer 54:337-352.

Maienfisch, P., M. Angst, F. Brandl, W. Fischer, D. Hofer, H. Kayser, et al. 2001 Chemistry and biology of thiamethoxam: a second generation neonicotinoid. Pest Manage. Sci. 57:906-913.

Mazzoni, E., and P. Cravedi. 2002. Analysis of insecticideresistant Myzus persicae (Sulzer) populations collected in Italian peach orchards. Pest Manage. Sci. 58:975-980.

McPherson, R.M., M.G. Stephenson, S.S. Lahue, and S.W. Mullis. 2005. Impact of early-season thrips management on reducing the risks of spotted wilt virus and suppressing aphid populations in flue-cured tobacco. J. Econ. Entomol. 98:129-134.

Nauen, R., and I. Denholm. 2005. Resistance of insect pests to neonicotinoid insecticides: current status and future prospects. Arch. Insect Biochem. Physiol. 58:200-215.

Nauen, R., and A. Elbert. 1997. Apparent tolerance of a field-collected strain of Myzus nicotianae to imidacloprid due to strong antifeeding responses. Pestic. Sci. 49:252-258.

Nauen, R., and A. Elbert. 2003. European monitoring of resistance to insecticides in Myzus persicae and Aphis gossypii (Hemiptera: Aphididae) with special reference to imidacloprid. Bull. Entomol. Res. 93:4754.
Nauen, R., H. Hungenberg, B. Tollo, K. Tietjen, and A. Elbert. 1998a. Antifeeding effect, biological efficacy and high affinity binding of imidacloprid to acetylcholine receptors in Myzus persicae and Myzus nicotianae. Pestic. Sci. 53:133-140.

Nauen, R., J. Strobel, K. Tietjen, Y. Otsu, C. Eldelen, and A. Elbert. 1996. Aphicidal activity of imidacloprid against a tobacco feeding strain of Myzus persicae (Homoptera: Aphididae) from Japan closely related to Myzus nicotianae and highly resistant to carbamates and organophosphates. Bull. Entomol. Res. 86:165171.

Nauen, R., K. Tietjen, K. Wagner, and A. Elbert. 1998b. Efficacy of plant metabolites of imidacloprid against Myzus persicae and Aphis gossypii. Pestic. Sci. 52:5357.

Sannino, L., F. Porrone, M.C. Biondani, M. Contiero, and C. Cersosimo. 2000a. Control of tobacco aphids by means of sprays. Il Tabacco 8:15-20.

Sannino, L., F. Porrone, M.C. Biondani, and G. Salerno. 2000b. Aphid control on Burley and Flue-cured tobacco with foliar insecticides. Il Tabacco 8:25-31.

Sarkar, M.A., S. Roy, R.K. Kole, and A. Chowdhury. 2001. Persistence and metabolism of imidacloprid in different soils of West Bengal. Pest Manage. Sci. 57:598-602.

Schmuck, R., R. Schöning, A. Stork, and O. Schramel. 2001. Risk posed to honeybees (Apis mellifera L., Hymenoptera) by an imidacloprid seed dressing of sunflowers. Pest Manage. Sci. 57:225-238.

Torres, J.B., C.S.A. Silva-Torres, and R. Barros. 2003. Relative effects of the insecticide thiamethoxam on the predator Podisus nigrispinus and the tobacco whitefly Bemisia tabaci in nectaried and nectariless cotton. Pest Manage. Sci. 59:315-323.

Unruh, T., A. Knight, and M.R. Bush. 1996. Green peach aphid (Homoptera: Aphididae) resistance to endosulfan in peach and nectarine orchards in Washington State. J. Econ. Entomol. 89:1067-1073.

Vincent, C., A. Ferran, L. Guige, J. Gambier, and J. Bru. 2000. Effects of imidacloprid on Harmonia axyridis (Coleoptera: Coccinellidae) larval biology and locomotory behavior. Eur. J. Entomol. 97:501-506.

Wolf, M.A., Y.A.I. Abdel-Aal, D.K.S. Goh, E.P. Lampert, and M. Roe. 1994. Organophosphate resistance in the tobacco aphid (Homoptera: Aphididae): purification and characterization of a resistance-associated esterase. J. Econ. Entomol. 87:1157-1164. 